\title{
Conservation Laws and Exact Solutions of Generalized Nonlinear System and Nizhink-Novikov-Veselov Equation
}

\author{
A. A. Zaidi, ${ }^{1}$ M. D. Khan $(1),{ }^{2}$ and I. Naeem $(1)^{1}$ \\ ${ }^{1}$ Department of Mathematics, Lahore University of Management Sciences, Lahore, Pakistan \\ ${ }^{2}$ Department of Mathematics and Statistics, Institute of Business Management, Karachi, Pakistan \\ Correspondence should be addressed to I. Naeem; imran.naeem@lums.edu.pk
}

Received 15 March 2018; Revised 6 June 2018; Accepted 15 August 2018; Published 25 September 2018

Academic Editor: Hang Xu

Copyright (c) 2018 A. A. Zaidi et al. This is an open access article distributed under the Creative Commons Attribution License, which permits unrestricted use, distribution, and reproduction in any medium, provided the original work is properly cited.

\begin{abstract}
The Lie symmetries, conservation laws, and exact solutions of a generalized nonlinear system and a (2+1)-dimensional generalized Nizhink-Novikov-Veselov (NNV) equation, arising in the study of hydrodynamics, are investigated. The multiplier approach is employed to compute the conservation laws for systems under consideration. The Lie point symmetries are derived and the association between symmetries and conserved vectors are established using symmetries conservation laws relationship. The double reduction theory is utilized which results in the reduction and exact solutions of models under investigation. All cases are discussed in detail and new solutions are determined.
\end{abstract}

\section{Introduction}

Noether [1] formulated a general approach for constructing conservation laws for Euler-Lagrange equations when LieBäcklund symmetries came to the fore. Noether's approach not only established a connection between conservation laws and symmetry properties of a physical system, but also provided a basis for the application of double reduction method. However, its applicability remained limited to those physical systems which have a Lagrangian formulation. Kara and Mahomed [2] relaxed this condition by introducing the concept of partial Lagrangian and associated Noether type symmetries with conservation laws. However, a connection between other types of symmetries and conservation laws remained elusive. Sjöberg $[3,4]$ developed the theory of double reduction for two independent variables. Bokhari et al. [5] then generalized this idea of reductions using conservation laws for $k$ independent variables.

Symmetries play an important role in many areas of physical sciences. In particular, hydrodynamics has remained an area of great interest owing to its applications in physics. Most of the studies have focused on providing an underlying mathematical theory by establishing empirical and semiempirical laws derived from the flow measurement of fluids and using them to solve real world problems. Mathematical models thus obtained provide vital information about various hydrological processes including the designing of dams, harbours, and bridges. These models usually entail systems of nonlinear partial differential equations (PDEs). Consequently, developing techniques for exact solutions is crucial to the analysis of such systems.

A system involving two nonlinear PDEs,

$$
\begin{aligned}
v_{t}(t, x)+a v(t, x) v_{x}(t, x)+b u_{x} & =0, \\
u_{t}(t, x)+a(u(t, x) v(t, x))_{x}+c v_{x x x}(t, x) & =0,
\end{aligned}
$$

where $a, b$, and $c$ are constants, arises in various physical phenomena in physics and engineering. As noted by Congy et al. [6], for $a=1, b=1$, and $c=-1 / 4$, the corresponding Kaup-Boussinesq (KB) system models the physics of shallow water waves. In this context, $u$ denotes the local height of the water layer and $v$ denotes the local mean flow velocity. The corresponding KB system is in fact a "positive dispersion" system, which also models the propagation of capillary waves on top of a thin fluid layer [7]. System (1) also serves as an approximation to various equations arising in physics and engineering. For instance, (1) provides an approximation to the Landau-Lifshitz equation, which models the propagation 
of magnetization waves in easy plane magnets. Similarly, the choice of coefficients $a=1, b=1$, and $c=1 / 3$ models a $(1+1)$-dimensional dispersive long wave equation [8], and the values $a=-2, b=-1, c=-1$ yield the Kaup-Boussinesq system [9]. System (1) also models the long wave phenomena in shallow water, where the depth of water is considered to be uniform and $u(t, x)$ and $v(t, x)$ denote the elevation of water waves and the surface velocity of water along $x$-direction, respectively. Further applications of (1) can be found in [1013] and some more recent works are given in [14] where soliton solutions are determined using Hirota's direct method and in [15] where symmetric analysis is used.

Another PDE that arises in the study of fluid dynamics is

$$
\begin{aligned}
u_{t} & +k u_{x x x}+r u_{y y y}+s u_{x}+q u_{y} \\
& =3 k\left(u \partial_{y}^{-1} u_{x}\right)_{x}+3 r\left(u \partial_{x}^{-1} u_{y}\right)_{y},
\end{aligned}
$$

where $\partial^{-1}$ denotes the integral with respect to the subscripts, $u=u(t, x, y)$ represents a physical field, and $k, s, q$, and $r$ are specified constants. The integro-partial differential equation (2) is the well-known generalized Nizhnik-NovikovVeselov (GNNV) equation [16, 17] and is essentially a symmetric generalization of the famous $\mathrm{KdV}$ equation to the $(2+1)$ dimensions [18], which has numerous applications in engineering and physics including the electrical transmission lines, surface gravity waves, internal solitons in the ocean, nonlinear acoustics of bubbly liquids, voidage slugs in fluidized beds, and the "Great Red spot" in Jupiter. For a detailed account of these applications, we refer the reader to [19]. Eq. (2) can be simplified using the transformations $u_{x}=v_{y}$ and $u_{y}=w_{x}$, where $v$ and $w$ are functions of $t, x$, and $y$. This yields the system

$$
\begin{aligned}
u_{t} & +k u_{x x x}+r u_{y y y}+s u_{x}+q u_{y} \\
& =3 k(u v)_{x}+3 r(u w)_{y}, \quad u_{x}=v_{y}, u_{y}=w_{x} .
\end{aligned}
$$

System (3) also arises in applications in plasma physics, optics, and condense matter physics and has been studied extensively. For instance, Boiti et al. [20] used inverse scattering method to procure solutions to (2), and Radha et al. [21] employed Painleve analysis and bilinear method to find multi-dromion solutions to (2). Some recent works on the GNNV equation can be found in $[22,23]$.

In this paper, we provide a double reduction analysis of the system of PDEs (1) and (3). The double reduction theory not only reduces the order and the number of independent variables involved but also makes use of symmetry, if it exists, to reduce a PDE further. Some of the solutions to (1) and (3) are of a "travelling wave" type and are based on a wellknown substitution, which is possible only if translational symmetries exist. In double reduction, not only are travelling wave type solutions obtained by making use of translational symmetries but also solutions are obtained by forming an association of the symmetry present with conserved vectors of the equation. This association is not only limited to the translational symmetries and it may exist for a wide range of symmetries like rotations, dilatations, inversions. Here, we exploit the dilation symmetry of the systems to extract new exact solutions. The interested reader is referred to some recent works in the field of symmetries, conservation laws, and the double reduction theory (see, e.g., $[24,25]$ ).

In Section 2, we provide a double reduction analysis of (1). We procure exact solutions to (1) by finding Lie symmetries and conservation laws. In Section 3, we extend this work to system (3). The results are discussed in Section 4. The conclusions are presented in Section 5.

\section{Generalized Nonlinear System}

We use the theory of double reduction to compute the exact solutions to (1) by first finding the conservation laws using the variational derivative approach. Many software packages are available to compute Lie symmetries of differential equations. For instance, the mathematical software Maple can be employed to obtain the Lie symmetries to (1). This yields

$$
\begin{aligned}
& X_{1}=\frac{\partial}{\partial t} \\
& X_{2}=\frac{\partial}{\partial x} \\
& X_{3}=a t \frac{\partial}{\partial x}+\frac{\partial}{\partial v} \\
& X_{4}=-2 t \frac{\partial}{\partial t}-x \frac{\partial}{\partial x}+2 u \frac{\partial}{\partial u}+v \frac{\partial}{\partial v} .
\end{aligned}
$$

We investigate the conservation laws of (1) using the multiplier approach. The multiplier $\Lambda_{\alpha}=\Lambda_{\alpha}\left(x, u, u_{(1)}, \ldots, u_{(\sigma)}\right)$ satisfies (see [26, 27])

$$
\Lambda_{\alpha}\left(\Delta^{\alpha}\right)=D_{i} T^{i}
$$

where $T$ is the conserved vector and

$$
\begin{aligned}
D_{i}=\frac{\partial}{\partial x^{i}}+u_{i}^{\theta} \frac{\partial}{\partial u^{\theta}}+u_{i j}^{\theta} \frac{\partial}{\partial u_{j}^{\theta}} & +\cdots, \\
& i=1, \cdots n, \theta=1, \cdots m,
\end{aligned}
$$

is the total derivative operator. The repeated indices throughout indicate the summation convention. The total derivative operator for two independent variables $(t, x)$ and two dependent variables $(u, v)$ takes the form

$$
\begin{aligned}
D_{t}= & \frac{\partial}{\partial t}+u_{t} \frac{\partial}{\partial u}+v_{t} \frac{\partial}{\partial v}+u_{t t} \frac{\partial}{\partial u_{t}}+u_{t x} \frac{\partial}{\partial u_{x}}+v_{t t} \frac{\partial}{\partial v_{t}} \\
& +v_{t x} \frac{\partial}{\partial v_{x}}+\ldots, \\
D_{x}= & \frac{\partial}{\partial x}+u_{x} \frac{\partial}{\partial u}+v_{x} \frac{\partial}{\partial v}+u_{x t} \frac{\partial}{\partial u_{t}}+u_{x x} \frac{\partial}{\partial u_{x}} \\
& +v_{x t} \frac{\partial}{\partial v_{t}}+v_{x x} \frac{\partial}{\partial v_{x}}+\ldots
\end{aligned}
$$

The variational derivative of (5) yields

$$
E_{u}\left[\Lambda_{\alpha}\left(\Delta^{\alpha}\right)\right]=0,
$$


where $E_{u}$ is the Euler operator and is given by

$$
\begin{aligned}
E_{u}=\frac{\partial}{\partial u^{\alpha}}+\sum_{s \geq 1}(-1)^{s} D_{i_{1}} \ldots D_{i_{s}} \frac{\partial}{\partial u_{i_{1} \ldots i_{s}}^{\alpha}} & \\
& \alpha=1,2, \ldots, m .
\end{aligned}
$$

The Euler operator $E_{u}$ for two independent variables $(t, x)$ and two dependent variables $(u, v)$ leads to

$$
\begin{aligned}
E_{u}= & \frac{\partial}{\partial u}-D_{t} \frac{\partial}{\partial u_{t}}-D_{x} \frac{\partial}{\partial u_{x}}+D_{t}^{2} \frac{\partial}{\partial u_{t t}}+D_{t} D_{x} \frac{\partial}{\partial u_{t x}} \\
& +D_{x}^{2} \frac{\partial}{\partial u_{x x}}-\ldots,
\end{aligned}
$$

where total derivative operators $D_{t}$ and $D_{x}$ are defined above.

$$
\text { Relation (8) gives }
$$

$$
\begin{aligned}
E_{u} & {\left[\Lambda_{1}\left(v_{t}+a v v_{x}+b u_{x}\right)+\Lambda_{2}\left(u_{t}+a(u v)_{x}+c v_{x x x}\right)\right] } \\
& =0 \\
E_{v} & {\left[\Lambda_{1}\left(v_{t}+a v v_{x}+b u_{x}\right)+\Lambda_{2}\left(u_{t}+a(u v)_{x}+c v_{x x x}\right)\right] } \\
& =0
\end{aligned}
$$

where $\Lambda_{1}=\Lambda_{1}(t, x, u, v)$ and $\Lambda_{2}=\Lambda_{2}(t, x, u, v)$. Equation (11) results in

$$
\begin{gathered}
\Lambda_{1 u}\left(v_{t}+a v v_{x}+b u_{x}\right)+\Lambda_{2 u}\left(u_{t}+a(u v)_{x}+c v_{x x x}\right) \\
+a \Lambda_{2} v_{x}-D_{x}\left(b \Lambda_{1}+a \Lambda_{2} v\right)-D_{t}\left(\Lambda_{2}\right)=0, \\
\Lambda_{1 v}\left(v_{t}+a v v_{x}+b u_{x}\right)+\Lambda_{2 v}\left(u_{t}+a(u v)_{x}+c v_{x x x}\right) \\
+a \Lambda_{1} v_{x}+\Lambda_{2}\left(a u_{x}\right)-D_{x}\left(a v \Lambda_{1}+a u \Lambda_{2}\right) \\
-D_{t}\left(\Lambda_{1}\right)+D_{x}^{3}\left(\Lambda_{2}\right)=0 .
\end{gathered}
$$

The separation of (12) with respect to the derivatives of $u$ and $v$ yields the system

$$
\begin{aligned}
\Lambda_{1 x} & =0, \\
\Lambda_{1 v} & =0, \\
\Lambda_{2 u} & =0, \\
\Lambda_{2 x x} & =0, \\
\Lambda_{2 x v} & =0, \\
\Lambda_{2 v v} & =0, \\
\Lambda_{1 u} & =\Lambda_{2 v}, \\
\Lambda_{1 t} & =-a u \Lambda_{2 x}, \\
\Lambda_{2 t} & =-a v \Lambda_{2 x},
\end{aligned}
$$

so that

$$
\begin{aligned}
& \Lambda_{1}(t, x, u, v)=-a t u c_{1}+u c_{2}+c_{4}, \\
& \Lambda_{2}(t, x, u, v)=(x-a t v) c_{1}+c_{2} v+c_{3},
\end{aligned}
$$

where $c_{1}, c_{2}, c_{3}$, and $c_{4}$ are arbitrary constants. Four sets of multipliers can be obtained by setting one of the constants equal to 1 and the rest to zero. This yields

$$
\begin{aligned}
& \Lambda_{1}^{1}(t, x, u, v)=-a t u, \\
& \Lambda_{2}^{1}(t, x, u, v)=x-a t v, \\
& \Lambda_{1}^{2}(t, x, u, v)=u, \\
& \Lambda_{2}^{2}(t, x, u, v)=v, \\
& \Lambda_{1}^{3}(t, x, u, v)=0, \\
& \Lambda_{2}^{3}(t, x, u, v)=1, \\
& \Lambda_{1}^{4}(t, x, u, v)=1, \\
& \Lambda_{2}^{4}(t, x, u, v)=0 .
\end{aligned}
$$

The conserved vectors corresponding to these multipliers are

$$
\begin{aligned}
T_{1}^{t}= & -a t u v+x u, \\
T_{1}^{x}= & -a^{2} t u v^{2}+a x u v-\frac{1}{2} a b t u^{2}-a c t v v_{x x}+\frac{1}{2} a c t v_{x}^{2} \\
& +c x v_{x x}-c v_{x}, \\
T_{2}^{t}= & u v, \\
T_{2}^{x}= & a u v^{2}+\frac{1}{2} b u^{2}+c v v_{x x}-\frac{1}{2} c v_{x}^{2}, \\
T_{3}^{t}= & u, \\
T_{3}^{x}= & a u v+c v_{x x}, \\
T_{4}^{t}= & v, \\
T_{4}^{x}= & \frac{1}{2} a v^{2}+b u .
\end{aligned}
$$

The above analysis shows that system (1) admits four symmetries and four conserved vectors.

We now compute the exact solutions of (1) by utilizing the double reduction theory. A Lie-Bäcklund symmetry generator $X$ is associated with a conserved vector $T$ of system (1) if it satisfies

$$
X\left(T^{i}\right)-T^{j} D_{j}\left(\xi^{i}\right)+T^{i} D_{j}\left(\xi^{j}\right)=0, \quad i=1,2, \ldots, n .
$$

The association between symmetry and conservation laws of system (1) is presented in Table 1.

Case 1 (solution of (1) using $X_{3}$ ). Let $X$ be any symmetry generator and $T$ be a conserved vector associated with $X$. Under a similarity transformation of $X$, there exists $\widetilde{T}$ such 
TABLE 1: Association between symmetries and conserved vectors.

\begin{tabular}{lcccc}
\hline Symmetries & $T_{1}$ & $T_{2}$ & $T_{3}$ & $T_{4}$ \\
\hline$X_{1}$ & NA & $\mathrm{A}$ & $\mathrm{A}$ & $\mathrm{A}$ \\
$X_{2}$ & NA & $\mathrm{A}$ & $\mathrm{A}$ & $\mathrm{A}$ \\
$X_{3}$ & $\mathrm{~A}$ & $\mathrm{NA}$ & $\mathrm{A}$ & $\mathrm{NA}$ \\
$X_{4}$ & $\mathrm{~A}$ & $\mathrm{NA}$ & $\mathrm{NA}$ & $\mathrm{A}$ \\
\hline
\end{tabular}

$\mathrm{A}=$ associated; $\mathrm{NA}=$ not associated.

that $\widetilde{D}_{j} \widetilde{T}^{j}=0$, where $\widetilde{T}$ can be calculated (see [5]) from the system

$$
\left(\begin{array}{c}
\widetilde{T}^{1} \\
\widetilde{T}^{2} \\
\vdots \\
\widetilde{T}^{n}
\end{array}\right)=\mathrm{J}\left(A^{-1}\right)^{T}\left(\begin{array}{c}
T^{1} \\
T^{2} \\
\vdots \\
T^{n}
\end{array}\right)
$$

where

$$
\begin{aligned}
A & =\left(\begin{array}{cccc}
\widetilde{D}_{1} x_{1} & \widetilde{D}_{1} x_{2} & \ldots & \widetilde{D}_{1} x_{n} \\
\widetilde{D}_{2} x_{1} & \widetilde{D}_{2} x_{2} & \ldots & \widetilde{D}_{2} x_{n} \\
\vdots & \vdots & \vdots & \vdots \\
\widetilde{D}_{n} x_{1} & \widetilde{D}_{n} x_{2} & \ldots & \widetilde{D}_{n} x_{n}
\end{array}\right), \\
A^{-1} & =\left(\begin{array}{cccc}
D_{1} \widetilde{x}_{1} & D_{1} \widetilde{x}_{2} & \ldots & D_{1} \widetilde{x}_{n} \\
D_{2} \widetilde{x}_{1} & D_{2} \widetilde{x}_{2} & \ldots & D_{2} \widetilde{x}_{n} \\
\vdots & \vdots & \vdots & \vdots \\
D_{n} \widetilde{x}_{1} & D_{n} \widetilde{x}_{2} & \ldots & D_{n} \widetilde{x}_{n}
\end{array}\right), \\
\mathrm{J} & =|A| .
\end{aligned}
$$

Relation (17) shows that the Lie symmetry $X_{3}$ is associated with $T_{1}$ and $T_{3}$. The change of variables

$$
\begin{aligned}
s & =\frac{x}{a t}, \\
r & =t, \\
A(r) & =\frac{-x+a t v}{a t}, \\
B(r) & =u .
\end{aligned}
$$

reduces the generator $X_{3}=a t(\partial / \partial x)+\partial / \partial v$ to the canonical form $\partial / \partial s$. For two independent variables, (18) reduces to the conserved form

$$
\left(\begin{array}{c}
T^{r} \\
T^{s}
\end{array}\right)=J\left(A^{-1}\right)^{T}\left(\begin{array}{c}
T^{t} \\
T^{x}
\end{array}\right)
$$

where

$$
\begin{aligned}
A^{-1} & =\left(\begin{array}{ll}
D_{t} r & D_{t} s \\
D_{x} r & D_{x} s
\end{array}\right), \\
J & =\operatorname{det}(A) .
\end{aligned}
$$

The $r$-components of $T_{1}$ and $T_{3}$ are obtained by exploiting relations (21) and (22). This gives

$$
\begin{aligned}
& T_{1}^{r}=-a^{2} r^{2} A B, \\
& T_{3}^{r}=a r B,
\end{aligned}
$$

so that

$$
\begin{aligned}
-a^{2} r^{2} A B & =g_{7}, \\
a r B & =g_{8},
\end{aligned}
$$

where $g_{7}$ and $g_{8}$ are constants. Equations (24) and (25) yield

$$
\begin{aligned}
& A(r)=\frac{-g_{7}}{g_{8} a r}, \\
& B(r)=\frac{g_{8}}{a r} .
\end{aligned}
$$

Consequently, the solution to (1) is

$$
\begin{aligned}
& u=\frac{g_{8}}{a t}, \\
& v=\frac{-g_{7}+x g_{8}}{a t g_{8}} .
\end{aligned}
$$

Case 2 (solution of (1) using $X_{1}$ and $X_{2}$ ). The symmetries $X_{1}$ and $X_{2}$ are associated with $T_{2}, T_{3}$, and $T_{4}$ (see Table 1). We derive the reduced conserved form by considering a combination of $X_{1}$ and $X_{2}$, namely, $X=\alpha X_{1}+X_{2}$. The generator $X$ has a canonical form $\partial / \partial s$ when

$$
\frac{d t}{\alpha}=\frac{d x}{1}=\frac{d u}{0}=\frac{d v}{0}=\frac{d r}{0}=\frac{d s}{1}=\frac{d w}{0}=\frac{d k}{0} .
$$

The characteristic equation (28) yields

$$
\begin{aligned}
r & =\alpha x-t, \\
s & =x, \\
w(r) & =u, \\
k(r) & =v .
\end{aligned}
$$

The conserved vectors $T_{2}, T_{3}$, and $T_{4}$, using (29) and (21)-(22), reduce to

$$
\begin{aligned}
& T_{2}^{r}=w k-\alpha a w k^{2}-\frac{1}{2} \alpha b w^{2}-\alpha^{3} c k k_{r r}+\frac{1}{2} c \alpha^{3} k_{r}^{2}, \\
& T_{2}^{s}=-a w k^{2}-\frac{1}{2} b w^{2}-\alpha^{2} c k k_{r r}-\frac{1}{2} c \alpha^{2} k_{r}^{2}, \\
& T_{3}^{r}=w-\alpha a w k-\alpha^{3} c k_{r r} \\
& T_{3}^{s}=-a w k+c \alpha^{2} k_{r r},
\end{aligned}
$$

$$
\begin{aligned}
T_{4}^{r} & =k-\frac{1}{2} \alpha a k^{2}-\alpha b w \\
T_{4}^{s} & =-\frac{1}{2} a k^{2}-b w .
\end{aligned}
$$


The conserved vectors $T_{2}^{r}, T_{3}^{r}$, and $T_{4}^{r}$ satisfy the reduced conserved form

$$
D_{r} T^{r}=0
$$

Consequently (30), (31), and (32) give

$$
\begin{aligned}
w k-\alpha a w k^{2}-\frac{1}{2} \alpha b w^{2}-\alpha^{3} c k k_{r r}+\frac{1}{2} c \alpha^{3} k_{r}^{2} & =d_{1}, \\
w-\alpha a w k-\alpha^{3} c k_{r r} & =d_{2}, \\
k-\frac{1}{2} \alpha a k^{2}-\alpha b w & =d_{3},
\end{aligned}
$$

where $d_{1}, d_{2}$, and $d_{3}$ are arbitrary constants. Any pair of equations in the reduced conserved form (34)-(36) can be used to construct a solution to (1) with the help of Maple. For instance, (34) and (35) give

$$
w(r)=\frac{d_{2}+\alpha^{3} c k_{r r}}{1-\alpha a k},
$$

and

$$
\begin{aligned}
& \pm \int 2 b c a \alpha^{3} e^{2 a \alpha c_{1}}\left[b c \left(1+8 b \alpha^{3} a^{2} d_{1} e^{4 a \alpha c_{1}}\right.\right. \\
& \quad \pm 4 a \alpha k e^{2 a \alpha c_{1}} \mp 2 a^{2} \alpha^{2} k^{2} e^{2 a \alpha c_{1}}+8 a^{2} b d_{2} \alpha^{3} k e^{4 a \alpha c_{1}} \\
& \quad+a^{4} \alpha^{4} k^{4} e^{4 a \alpha c_{1}}-4 a \alpha k e^{4 a \alpha c_{1}}+6 a^{2} \alpha^{2} k^{2} e^{4 a \alpha c_{1}} \\
& \left.\left.\quad-4 a^{3} \alpha^{3} k^{3} e^{4 a \alpha c_{1}}+e^{4 a \alpha c_{1}} \mp 2 e^{2 a \alpha c_{1}}\right)\right]^{-1 / 2} d k \\
& \quad-r-g_{2}=0 .
\end{aligned}
$$

Another exact solution to (1) can be gleaned using (34) and (36). This gives

$$
\begin{aligned}
& k_{1}(r)=\frac{1}{a \alpha}(1 \\
& \left.+\tan \left(\frac{\sqrt{2 a \alpha d_{3}-1}}{2 \alpha^{2} \sqrt{b c}}\left(r+c_{1}\right)\right) \sqrt{2 a \alpha d_{3}-1}\right), \\
& w_{1}(r)=\frac{-1}{\alpha^{2} b a}\left(a \alpha d_{3}-\frac{1}{2}\right) \\
& \cdot \sec ^{2}\left(\frac{\sqrt{2 a \alpha d_{3}-1}}{2 \alpha^{2} \sqrt{b c}}\left(r+c_{1}\right)\right),
\end{aligned}
$$

and

$$
\begin{aligned}
& k_{2}(r)=\frac{1}{a \alpha}(1 \\
& \left.-\tan \left(\frac{\sqrt{2 a \alpha d_{3}-1}}{2 \alpha^{2} \sqrt{b c}}\left(r+c_{1}\right)\right) \sqrt{2 a \alpha d_{3}-1}\right), \\
& w_{2}(r)=\frac{1-2 a \alpha d_{3}}{2 \alpha^{2} b a} \sec ^{2}\left(\frac{\sqrt{2 a \alpha d_{3}-1}}{2 \alpha^{2} \sqrt{b c}}\left(r+c_{1}\right)\right), \\
& d_{1}=0 .
\end{aligned}
$$

It can be verified directly that solutions (39) and (40) satisfy (1). The same solution is obtained from (35) and (36) by setting $d_{2}=0$ and solving the equations simultaneously.

The transformation $r=\alpha x-t$ introduced in (29) describes one-dimensional travelling wave, where $\alpha$ is the wave number and $1 / \alpha$ is the wave speed. If we choose $a=$ $1, b=1$, and $c=1 / 3$, then system (1) is identified as dispersive long wave system [8]. Here $a$ is a nonlinearity parameter and $b$ is dispersive parameter. If we set $1 / \alpha>2 a d_{3}$, then the exact solution (39) and (40) corresponds for internal waves. If the wave speed $1 / \alpha$ is chosen to be 1.01 and $d_{3}=$ -0.51 , then the elevation in (39) of waves is shown in Figure 1.

If $1 / \alpha<2 a d_{3}$, then the solution (39) represents the rogue waves. This situation is depicted in Figure 2, where we assume $\alpha=1$ and $d_{3}=1$.

The elevation function $u(t, x)$ of (39) is the same as (40).

Case 3 (solution of (1) using $X_{4}$ ). Relation (17) shows that the symmetry $X_{4}$ is associated with $T_{1}$ and $T_{4}$. Canonical coordinates of $X_{4}$ are

$$
\begin{aligned}
r & =\frac{x}{\sqrt{t}}, \\
s & =-\frac{1}{2} \ln (t), \\
A(r) & =v \sqrt{t}, \\
B(r) & =t u .
\end{aligned}
$$

Equations (21) and (22) along with (41) can be used to procure the reduced conserved form of vectors $T_{1}$ and $T_{4}$. This yields

$$
\begin{aligned}
3 a r A B & -r^{2} B-2 a^{2} A^{2} B-a b B^{2}-2 a c A A_{r r}+a c A_{r}^{2} \\
& +2 c r A_{r r}-2 c A_{r}=g_{9},
\end{aligned}
$$

and

$$
-r A+a A^{2}+2 b B=g_{10} .
$$

Since (42) and (43) do not admit any symmetry, the double reduction theory can not be applied to find the exact solution of (1). However, numerical methods can be employed to obtain approximate solutions (Figure 3).

\section{3. (2+1)-Dimensional GNNV Equation}

In this section, we compute exact solutions to the $(2+1)$ dimensional system (3) by following a pattern similar to that used in Section 2. This gives the Lie point symmetries admitted by (3)

$$
\begin{aligned}
& X_{1}=\frac{\partial}{\partial t}+G_{1}(t) \frac{\partial}{\partial x}-\frac{1}{3 k} G_{1}^{\prime}(t) \frac{\partial}{\partial v} \\
& X_{2}=\frac{\partial}{\partial y}+G_{2}(t) \frac{\partial}{\partial x}-\frac{1}{3 k} G_{2}^{\prime}(t) \frac{\partial}{\partial v} \\
& X_{3}=t \frac{\partial}{\partial t}+\left(\frac{x}{3}+G_{3}(t)\right) \frac{\partial}{\partial x}+\frac{y}{3} \frac{\partial}{\partial y}-\frac{2 u}{3} \frac{\partial}{\partial u}
\end{aligned}
$$




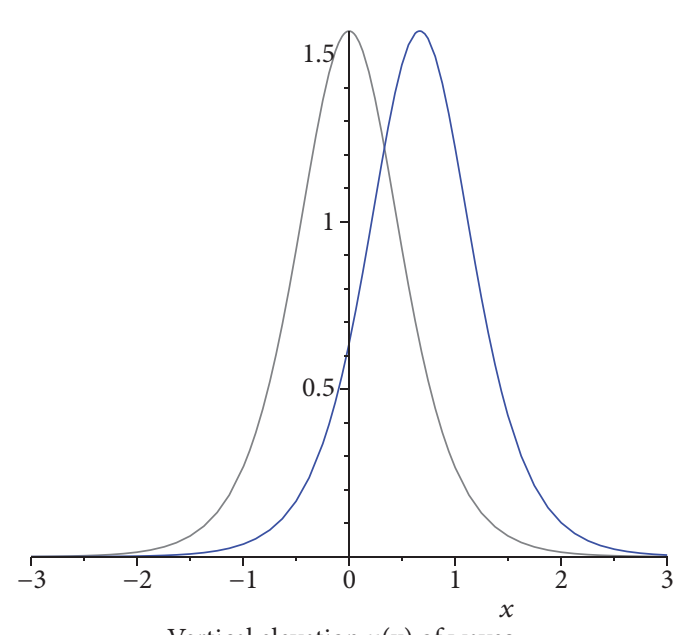

Vertical elevation $\mathrm{u}(\mathrm{x})$ of waves

$\mathrm{t}=0$

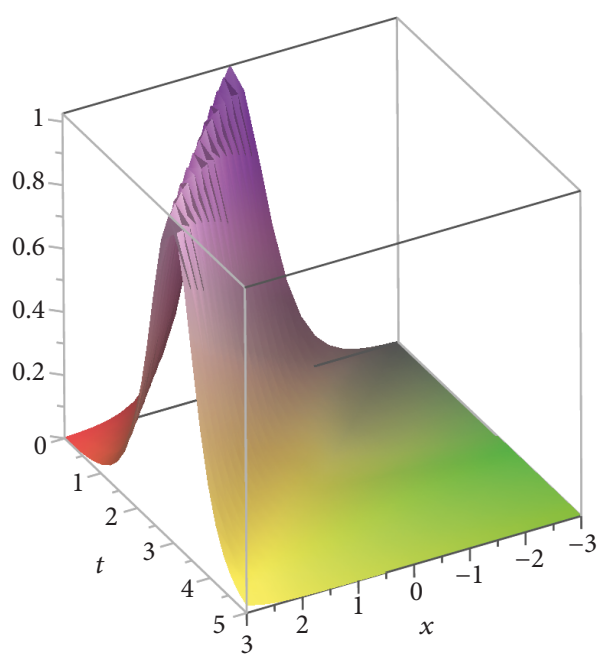

Vertical elevation $\mathrm{u}(\mathrm{t}, \mathrm{x})$

Figure 1
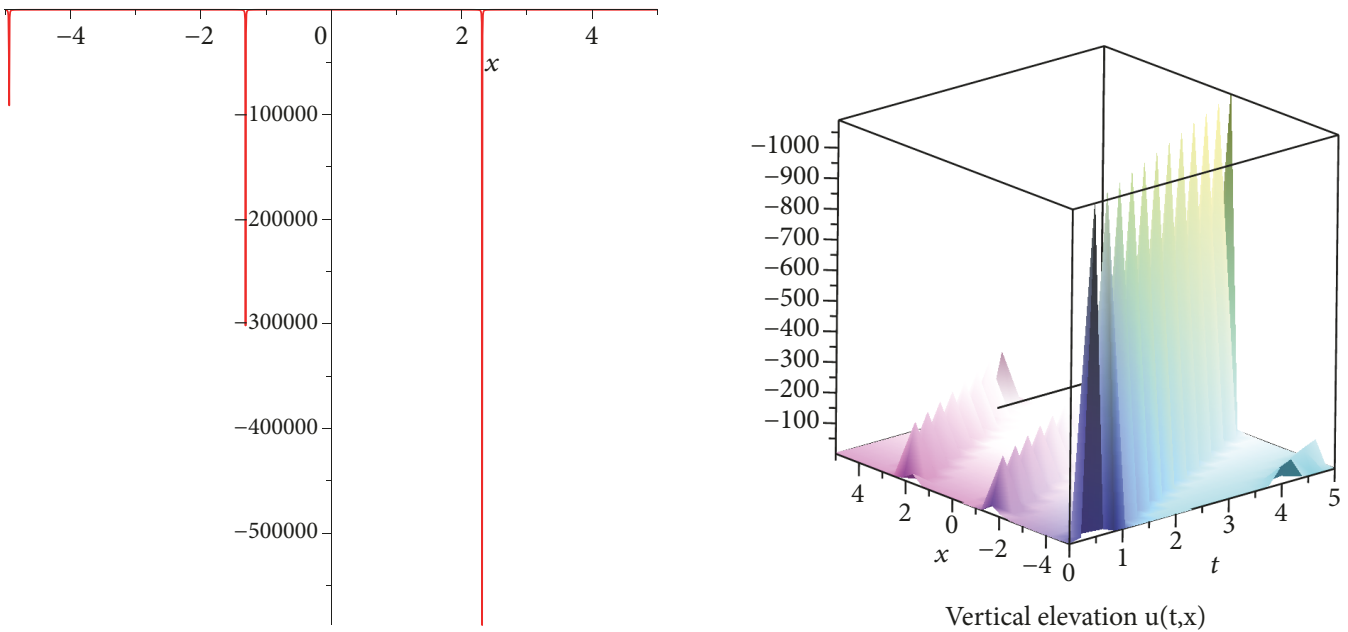

Vertical elevation $\mathrm{u}(\mathrm{t}, \mathrm{x})$

Vertical elevation $\mathrm{u}(\mathrm{x})$

FiguRE 2
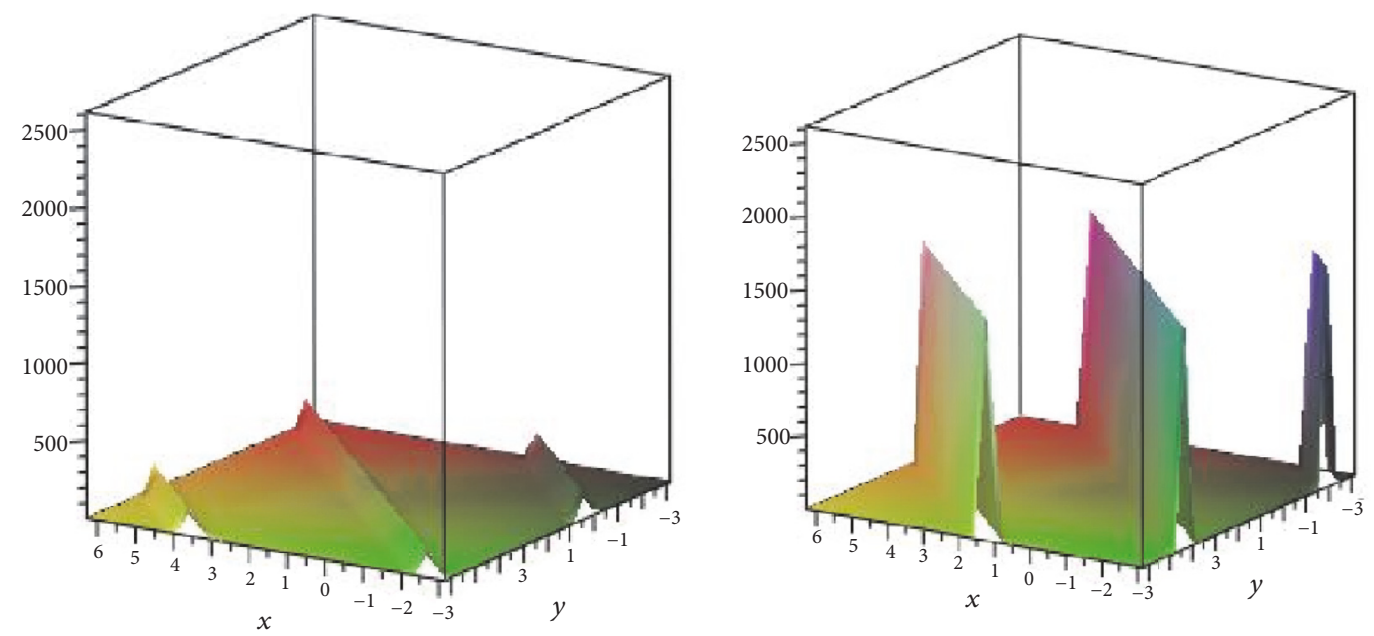

FIGURE 3: Solution profile of $M_{1}$ where $k=1, r=1, s=-1, q=1, \gamma=-1, \alpha=-1, \beta=-1$. This sequence of frames is constructed from time $t=0$ to $t=10$. 


$$
\begin{aligned}
& +\frac{1}{9 k}\left(-3 G_{3}^{\prime}(t)-6 k v+2 s\right) \frac{\partial}{\partial v} \\
& +\frac{1}{9 r}(2 q-6 r w) \frac{\partial}{\partial w}
\end{aligned}
$$

and the following set of determining equations for multipliers $\Lambda_{k}=\Lambda_{k}(t, x, u, v, w)$, where $k=1,2,3$

$$
\begin{aligned}
& \Lambda_{2 x x}=0 \text {, } \\
& \Lambda_{3 y y}=0 \text {, } \\
& \Lambda_{1 t}=-\Lambda_{2 x}-\Lambda_{3 y}, \\
& \Lambda_{1 x}=0 \text {, } \\
& \Lambda_{3 x}=0 \text {, } \\
& \Lambda_{1 y}=0 \text {, } \\
& \Lambda_{2 y}=0 \text {, } \\
& \Lambda_{1 u x}=0 \text {, } \\
& \Lambda_{1 u}=0 \text {, } \\
& \Lambda_{2 u}=0 \text {, } \\
& \Lambda_{3 u}=0 \text {, } \\
& \Lambda_{1 v}=0 \text {, } \\
& \Lambda_{2 v}=0 \text {, } \\
& \Lambda_{3 v}=0 \text {, } \\
& \Lambda_{1 w}=0, \\
& \Lambda_{2 w}=0 \text {, } \\
& \Lambda_{3 w}=0 \text {, } \\
& \Lambda_{2 u x}=0 \text {, } \\
& \Lambda_{3 u x}=0 \text {, } \\
& \Lambda_{1 v x}=0 \text {, } \\
& \Lambda_{2 v x}=0 \text {, } \\
& \Lambda_{3 v x}=0 \text {, } \\
& \Lambda_{1 w x}=0 \text {, } \\
& \Lambda_{2 w x}=0 \text {, } \\
& \Lambda_{3 w x}=0 .
\end{aligned}
$$

The last set of equations give

$$
\begin{aligned}
& \Lambda_{1}=-F_{1}(t)-F_{3}(t)+c_{1}, \\
& \Lambda_{2}=F_{1}(t) x+F_{2}(t), \\
& \Lambda_{3}=F_{3}(t) y+F_{4}(t),
\end{aligned}
$$

so that the conserved vectors corresponding to these multipliers are

$$
\begin{aligned}
T_{1}^{t} & =u, \\
T_{1}^{x} & =-3 u k v+u s+u_{x x} k, \\
T_{1}^{y} & =-3 u r w+u q+u_{y y} r, \\
T_{2}^{t} & =-u F_{1}(t), \\
T_{2}^{x} & =3 u F_{1}(t) k v-u F_{1}(t) s-u_{x x} F_{1}(t) k+u F_{1}^{\prime}(t) x, \\
T_{2}^{y} & =3 u F_{1}(t) r w-u F_{1}(t) q-u_{y y} F_{1}(t) r-v F_{1}^{\prime}(t) x, \\
T_{3}^{t} & =0, \\
T_{3}^{x} & =u F_{2}(t), \\
T_{3}^{y} & =-v F_{2}(t), \\
T_{4}^{t} & =-u F_{3}(t), \\
T_{4}^{x} & =3 u F_{3}(t) k v-u F_{3}(t) s-w F_{3}^{\prime}(t) y-u_{x x} F_{3}(t) k, \\
T_{4}^{y} & =3 u F_{3}(t) r w-u F_{3}(t) q+u F_{3}^{\prime}(t) y-u_{y y} F_{3}(t) r, \\
T_{5}^{t} & =0 \\
T_{5}^{x} & =-w F_{4}(t), \\
T_{5}^{y} & =u F_{4}(t) .
\end{aligned}
$$

The association between symmetries and conservation laws is shown in Table 2.

Case 1 (solutions of (3) using $X_{1}$ and $X_{2}$ ). The Lie symmetries $X_{1}$ and $X_{2}$ are associated with the conserved vectors $T_{1}, T_{2}$, $T_{3}, T_{4}$, and $T_{5}$ if $F_{i}(t)$ and $G_{j}(t)$ are constants. For simplicity, we assume these constants to be equal to 1 . Consequently, the Lie symmetries reduce to

$$
\begin{aligned}
& X_{1}=\frac{\partial}{\partial t}+\frac{\partial}{\partial x} \\
& X_{2}=\frac{\partial}{\partial x}+\frac{\partial}{\partial y}
\end{aligned}
$$

and the associated conserved vectors take the form

$$
\begin{aligned}
& T_{1}^{t}=u, \\
& T_{1}^{x}=-3 k u v+s u+k u_{x x}, \\
& T_{1}^{y}=-3 r u w+q u+r u_{y y}, \\
& T_{2}^{t}=-u, \\
& T_{2}^{x}=3 k u v-s u-k u_{x x}, \\
& T_{2}^{y}=3 r u w-q u-r u_{y y}, \\
& T_{3}^{t}=0,
\end{aligned}
$$




$$
\begin{aligned}
& T_{3}^{x}=u, \\
& T_{3}^{y}=-v, \\
& T_{4}^{t}=-u, \\
& T_{4}^{x}=3 k u v-s u-k u_{x x}, \\
& T_{4}^{y}=3 r u w-q u-r u_{y y}, \\
& T_{5}^{t}=0, \\
& T_{5}^{x}=-w, \\
& T_{5}^{y}=u .
\end{aligned}
$$

We note that the conserved vectors $T_{1}, T_{2}$, and $T_{4}$ are the same, so we only use $T_{1}$ to construct the solution of (3). A linear combination of $X_{1}$ and $X_{2}$, given in (48), can be expressed as

$$
X=\frac{\partial}{\partial t}+\alpha \frac{\partial}{\partial x}+\beta \frac{\partial}{\partial y}
$$

where $\alpha$ and $\beta$ are constants. The generator $X$ in the last equation reduces to $\partial / \partial c$ with the help of similarity transformations

$$
\begin{aligned}
a & =y-\beta t, \\
b & =x-\alpha t, \\
c & =t, \\
u(t, x, y) & =A(a, b), \\
v(t, x, y) & =B(a, b), \\
w(t, x, y) & =C(a, b) .
\end{aligned}
$$

Equation (18) gives

$$
\left(\begin{array}{c}
T^{a} \\
T^{b} \\
T^{c}
\end{array}\right)=J\left(A^{-1}\right)^{T}\left(\begin{array}{c}
T^{t} \\
T^{x} \\
T^{y}
\end{array}\right),
$$

where

$$
J=-1
$$

$$
\text { and }\left(A^{-1}\right)^{T}=\left(\begin{array}{ccc}
\beta & 0 & -1 \\
\alpha & -1 & 0 \\
-1 & 0 & 0
\end{array}\right) \text {. }
$$

This yields

$$
\begin{aligned}
& T^{a}=\beta T^{t}-T^{y}, \\
& T^{b}=\alpha T^{t}-T^{x}, \\
& T^{c}=-T^{t},
\end{aligned}
$$

so that the reduced conserved vectors are

$$
\begin{aligned}
& T_{1}^{a}=\beta A+3 r A C-q A-r A_{a a}, \\
& T_{1}^{b}=\alpha A+3 k A B-s A-k A_{b b}, \\
& T_{3}^{a}=B, \\
& T_{3}^{b}=-A,
\end{aligned}
$$

and

$$
\begin{aligned}
& T_{5}^{a}=-A, \\
& T_{5}^{b}=C .
\end{aligned}
$$

We note that the new conservation law satisfies

$$
D_{a} T^{a}+D_{b} T^{b}=0
$$

The transformed vectors $T_{1}, T_{3}$, and $T_{5}$ in (55)-(57) inherit symmetries $\partial / \partial a$ and $\partial / \partial b$ which are helpful in further reduction. The combination of these symmetries $\widetilde{X}=\partial / \partial a+$ $\gamma(\partial / \partial b)$ yields the similarity variables

$$
\begin{aligned}
m & =a, \\
n & =-\gamma a+b, \\
L(n) & =C(a, b), \\
M(n) & =A(a, b), \\
N(n) & =B(a, b),
\end{aligned}
$$

and (19) can be used to procure

$$
\begin{aligned}
& T^{n}=\gamma T^{a}-T^{b}, \\
& T^{m}=-T^{a},
\end{aligned}
$$

which satisfies

$$
D_{n} T^{n}=0
$$

Integrating the last equation yields

$$
T^{n}=d,
$$

where $d$ is the constant of integration. The conserved vectors (55)-(57) using (60) and (62) can be expressed as

$$
\begin{aligned}
& \gamma \beta M+3 r \gamma M L-q \gamma M-r \gamma^{3} M_{n n}-\alpha M-3 k M N \\
& \quad+s M+k M_{n n}=d_{1}, \\
& \gamma N+M=d_{2}, \\
& \gamma M+L=d_{3},
\end{aligned}
$$


where $d_{1}, d_{2}$, and $d_{3}$ are arbitrary constants. The solution of system (63) is

$$
\begin{aligned}
& N=\frac{d_{2}-M}{\gamma}, \\
& L=d_{3}-\gamma M \text {, } \\
& \pm \int\left(\gamma ( r \gamma ^ { 3 } - k ) \left(\gamma^{2} \beta M^{2}+3 \gamma^{2} r d_{3} M^{2}-2 \gamma^{3} r M^{3}\right.\right. \\
& -\gamma^{2} q M^{2}-\alpha \gamma M^{2}-3 k d_{2} M^{2}+2 k M^{3}+s \gamma M^{2} \\
& \left.\left.+c_{1} r \gamma^{4}-c_{1} \gamma k-2 d_{1} \gamma M\right)\right)^{-1 / 2} d M-n \\
& -c_{2}=0 \text {, }
\end{aligned}
$$

$$
n=(\beta \gamma-\alpha) t-\gamma y+x
$$

It can be verified directly that these solutions satisfy (3). Explicit solutions to (63) can be procured by setting constants $d_{1}, d_{2}$, and $d_{3}$ equal to zero and then employing the sinecosine method [28]. This gives

$$
\begin{gathered}
M_{1}(n)=-\frac{1}{2} \frac{\gamma(\alpha+q \gamma-\gamma \beta-s)}{r \gamma^{3}-k} \\
\cdot \sec ^{2}\left(\frac{1}{2} \sqrt{\frac{-\gamma \beta-s+q \gamma+\alpha}{r \gamma^{3}-k}} n\right), \\
N_{1}(n)=\frac{1}{2} \frac{(\alpha+q \gamma-\gamma \beta-s)}{r \gamma^{3}-k} \\
\cdot \sec ^{2}\left(\frac{1}{2} \sqrt{\frac{-\gamma \beta-s+q \gamma+\alpha}{r \gamma^{3}-k}} n\right), \\
L_{1}(n)=\frac{1}{2} \frac{\gamma^{2}(\alpha+q \gamma-\gamma \beta-s)}{r \gamma^{3}-k} \\
\cdot \sec ^{2}\left(\frac{1}{2} \sqrt{\frac{-\gamma \beta-s+q \gamma+\alpha}{r \gamma^{3}-k}} n\right), \\
\left.\frac{-\gamma \beta-s+q \gamma+\alpha}{r \gamma^{3}-k} n\right) \\
\left.M_{2}(n)=-\frac{1}{2} \frac{\gamma(\alpha+q \gamma-\gamma \beta-s)}{r \gamma^{3}-k} \sqrt{\frac{-\gamma \beta-s+q \gamma+\alpha}{r \gamma^{3}-k}}\right)
\end{gathered}
$$

$$
\begin{aligned}
& N_{2}(n)=\frac{1}{2} \frac{(\alpha+q \gamma-\gamma \beta-s)}{r \gamma^{3}-k} \\
& \cdot \csc ^{2}\left(\frac{1}{2} \sqrt{\frac{-\gamma \beta-s+q \gamma+\alpha}{r \gamma^{3}-k}} n\right), \\
& L_{2}(n)=\frac{1}{2} \frac{\gamma^{2}(\alpha+q \gamma-\gamma \beta-s)}{r \gamma^{3}-k} \\
& \cdot \csc ^{2}\left(\frac{1}{2} \sqrt{\frac{-\gamma \beta-s+q \gamma+\alpha}{r \gamma^{3}-k}} n\right), \\
& \frac{-\gamma \beta-s+q \gamma+\alpha}{r \gamma^{3}-k}>0 .
\end{aligned}
$$

$$
\begin{gathered}
M_{3}(n)=-\frac{1}{2} \frac{\gamma(\alpha+q \gamma-\gamma \beta-s)}{r \gamma^{3}-k} \\
\cdot \operatorname{sech}^{2}\left(\frac{1}{2} \sqrt{\frac{\gamma \beta+s-q \gamma-\alpha}{r \gamma^{3}-k}} n\right),
\end{gathered}
$$$$
N_{3}(n)=\frac{1}{2} \frac{(\alpha+q \gamma-\gamma \beta-s)}{r \gamma^{3}-k}
$$$$
\cdot \operatorname{sech}^{2}\left(\frac{1}{2} \sqrt{\frac{\gamma \beta+s-q \gamma-\alpha}{r \gamma^{3}-k}} n\right) \text {, }
$$

$L_{3}(n)=\frac{1}{2} \frac{\gamma^{2}(\alpha+q \gamma-\gamma \beta-s)}{r \gamma^{3}-k}$

$$
\cdot \operatorname{sech}^{2}\left(\frac{1}{2} \sqrt{\frac{\gamma \beta+s-q \gamma-\alpha}{r \gamma^{3}-k}} n\right) \text {, }
$$$$
\frac{-\gamma \beta-s+q \gamma+\alpha}{r \gamma^{3}-k}<0 \text {. }
$$$$
M_{4}(n)=\frac{1}{2} \frac{\gamma(\alpha+q \gamma-\gamma \beta-s)}{r \gamma^{3}-k}
$$$$
\cdot \operatorname{csch}^{2}\left(\frac{1}{2} \sqrt{\frac{\gamma \beta+s-q \gamma-\alpha}{r \gamma^{3}-k}} n\right) \text {, }
$$$$
N_{4}(n)=-\frac{1}{2} \frac{(\alpha+q \gamma-\gamma \beta-s)}{r \gamma^{3}-k}
$$$$
\cdot \operatorname{csch}^{2}\left(\frac{1}{2} \sqrt{\frac{\gamma \beta+s-q \gamma-\alpha}{r \gamma^{3}-k}} n\right) \text {, }
$$

$$
\begin{aligned}
& L_{4}(n)=-\frac{1}{2} \frac{\gamma^{2}(\alpha+q \gamma-\gamma \beta-s)}{r \gamma^{3}-k} \\
& \cdot \operatorname{csch}^{2}\left(\frac{1}{2} \sqrt{\frac{\gamma \beta+s-q \gamma-\alpha}{r \gamma^{3}-k}} n\right), \\
& \frac{-\gamma \beta-s+q \gamma+\alpha}{r \gamma^{3}-k}<0 .
\end{aligned}
$$



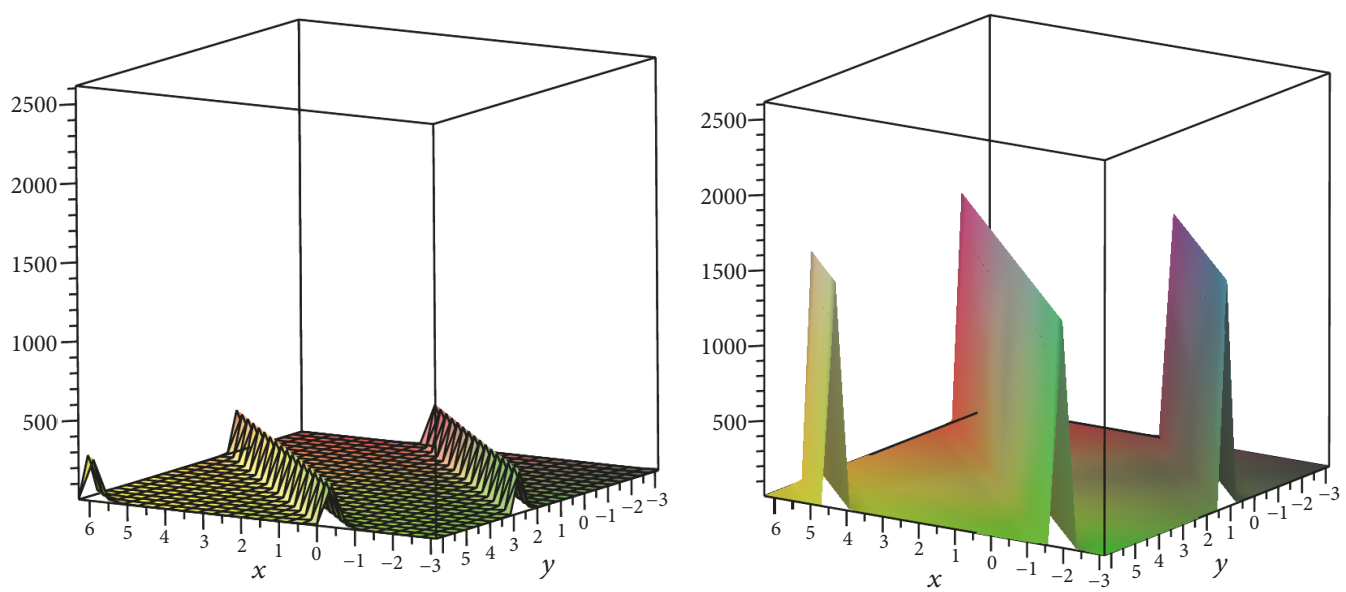

FIGURE 4: Solution profile $M_{2}$ where $k=1, r=1, s=-1, q=1, \gamma=-1, \alpha=-1, \beta=-1$. This sequence of frames is constructed from time $t=0$ to $t=10$.

TABLE 2: Association between symmetries and conservation laws.

\begin{tabular}{ccccc}
\hline & $T_{1}$ & $T_{2}$ & $T_{3}$ & $T_{4}$ \\
\hline$X_{1}$ & associated & associated if & associated if & associated if \\
& $F_{1}(t)$ constant & $F_{2}(t)$ and $G_{1}(t)$ constant & $F_{3}(t)$ constant & associated if \\
& associated & associated if & associated if & associated if \\
& $F_{1}(t)$ constant & $F_{2}(t)=0, G_{2}(t) \neq 0$ & $F_{3}(t)$ constant \\
& or $G_{2}(t)=0$ & or $F_{2}(t) \neq 0, G_{2}(t)$ constant & \\
\hline$X_{2}$ & associated if & associated if & associated if \\
& associated & $F_{1}(t)$ constant & $G_{3}(t)=2 s t / 3, F_{2}(t)=t^{-2 / 3}$ & $F_{3}(t)$ constant \\
\hline
\end{tabular}

In system (3), $u$ represents physical field and $v$ and $w$ describe potentials. We recover solution $\left(M_{3}, N_{3}, L_{3}\right)$ in (68) as reported in [17]. This solution is identified as dromion solution. Here, we obtain three additional solutions $\left(M_{1}, N_{1}, L_{1}\right)$, $\left(M_{2}, N_{2}, L_{2}\right)$, and $\left(M_{4}, N_{4}, L_{4}\right)$ of system (3) which were not reported before and their profiles are shown below.

Figure 4 represents the breather wave.

Case 2 (solution of (3) using $X_{3}$ ). As shown in Table 2, the Lie symmetry $X_{3}$ is associated with the conserved vectors $T_{1}$ and $T_{3}$ if $F_{2}(t)=t^{-2 / 3}$ and $G_{3}(t)=(2 / 3) s t$. This leads to the similarity transformations of $X_{3}$, which are given by

$$
\begin{aligned}
a & =\frac{x-s t}{t^{1 / 3}}, \\
b & =\frac{y}{t^{1 / 3}}, \\
c & =\ln (t), \\
A(a, b) & =v t^{2 / 3}, \\
B(a, b) & =-\frac{1}{3 r} t^{2 / 3}(-3 r w+q), \\
C(a, b) & =u t^{2 / 3} .
\end{aligned}
$$

The conserved vectors $T_{1}$ and $T_{3}$ in new variables can be expressed as

$$
\begin{aligned}
T_{1}^{a} & =-\frac{1}{3} a C-3 k A C+k C_{a a}, \\
T_{1}^{b} & =-\frac{1}{3} b C-3 r B C+r C_{b b}, \\
T_{3}^{a} & =C \\
T_{3}^{b} & =-A .
\end{aligned}
$$

The above vectors are invariant under Lie symmetry $9 r(\partial / \partial b)-\partial / \partial B$. Consequently, the similarity variables are

$$
\begin{aligned}
m & =\frac{b}{9 r}, \\
n & =a, \\
L(n) & =C(a, b), \\
M(n) & =A(a, b), \\
N(n) & =\frac{1}{9 r}(b+9 r B),
\end{aligned}
$$

so that

$$
\begin{aligned}
& T^{n}=9 r T^{a}, \\
& T^{m}=T^{b} .
\end{aligned}
$$



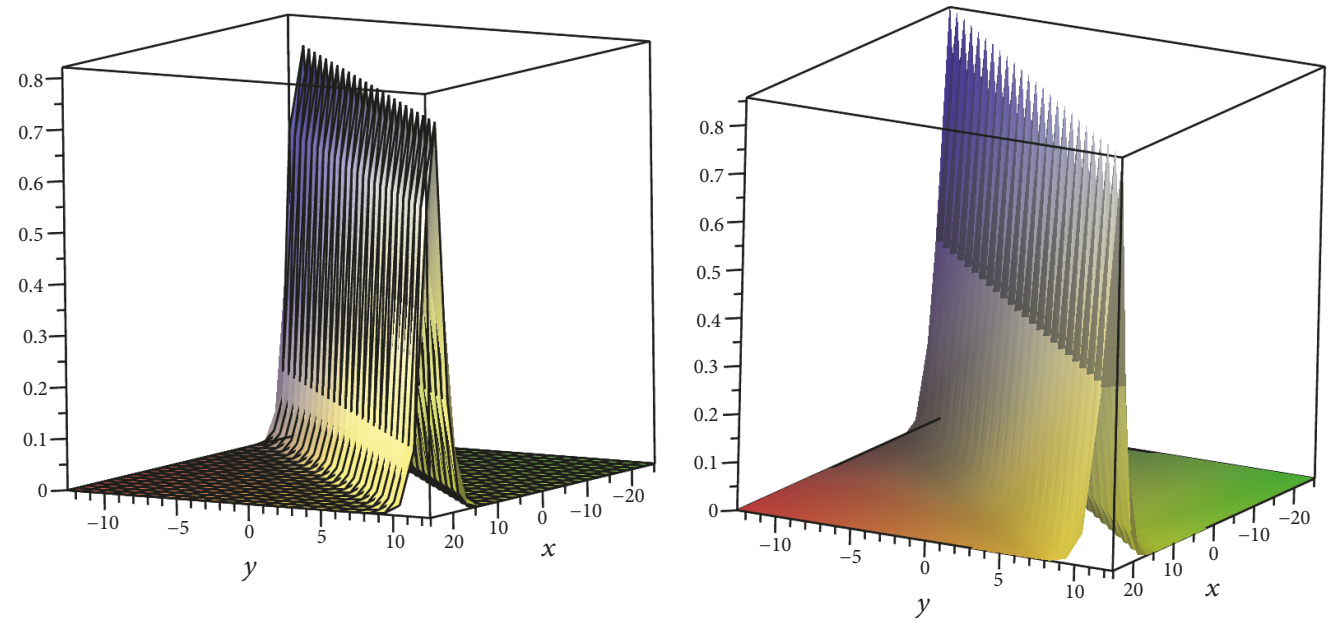

Figure 5: Solution profile $M_{3}$ where $k=1, r=1, s=-1, q=1, \gamma=2, \alpha=-1, \beta=4$. This sequence of frames is constructed for time $t=0$ to $t=10$.
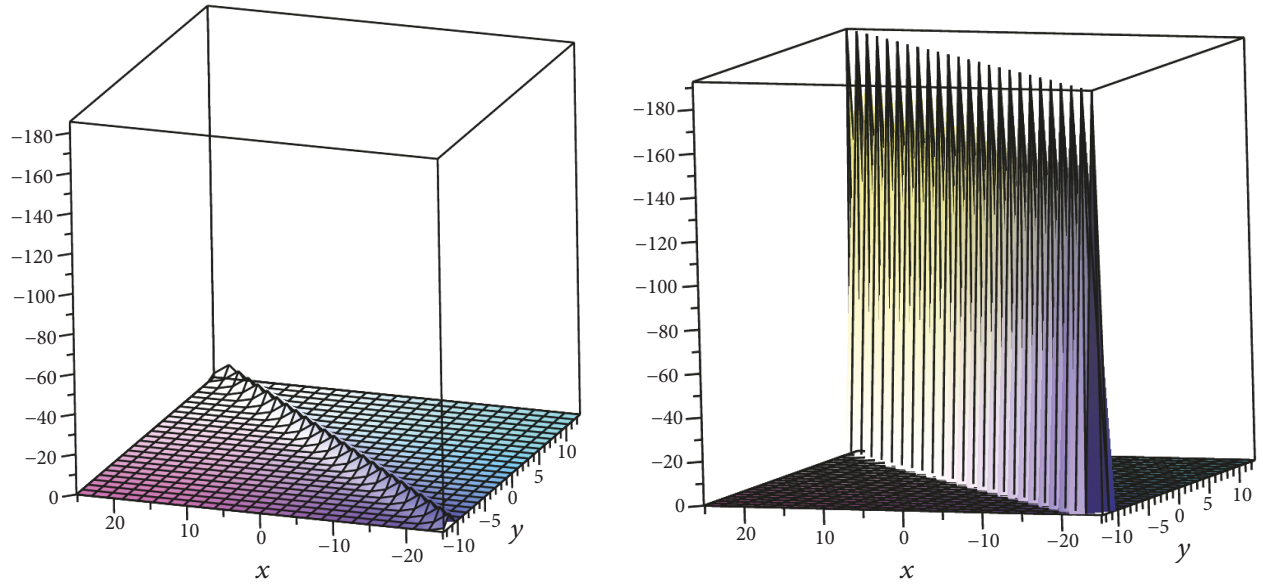

FIGURE 6: Solution profile $M_{4}$ where $k=1, r=1, s=-1, q=1, \gamma=2, \alpha=-1, \beta=4$. This sequence of frames is constructed for time $t=0$ to $t=2$.

The reduced conserved form thus gives

$$
\begin{aligned}
-3 r n L-27 r k M L+k L_{n n} & =d_{7}, \\
9 r L & =d_{8},
\end{aligned}
$$

where we have replaced $T^{n}$ by $d_{7}$ and $T^{m}$ by $d_{8}$. We note that the above system does not have any contribution from the third equation of (3), namely, $u_{y}-w_{x}=0$ (Figures 5 and 6). This equation is invariant under $X_{3}$, and the change of variables (70) yields

$$
C_{b}=B_{a} .
$$

Since (75) is also invariant under the symmetry $9 r(\partial / \partial b)-$ $\partial / \partial B$, its further reduction leads to

$$
N(n)=d_{9} .
$$

Equations (76) and (74) in original variables give the solution of (3) (see Figures 7-9)

$$
\begin{aligned}
& u=\frac{d_{8}}{9 r t^{2 / 3}}, \\
& v=\frac{1}{3 k d_{8} t^{2 / 3}}\left(\frac{d_{8}(s t-x)}{3 t^{1 / 3}}-d_{7}\right), \\
& w=\frac{1}{9 r t^{2 / 3}}\left(9 r d_{9}+3 q t^{2 / 3}-y t^{-1 / 3}\right) .
\end{aligned}
$$

We note from Table 2 that $T_{2}$ and $T_{4}$ are also associated with $X_{3}$ if $F_{1}(t)$ and $F_{3}(t)$ are constants but in that case $T_{2}$ and $T_{4}$ reduce to $T_{1}$ which has already been discussed above.

Case 3 (solution of (3) using $X_{1}, X_{2}, T_{1}$, and $T_{5}$ ). Consider $X=X_{1}+\alpha X_{2}$. As shown in Table 2, the symmetries $X_{1}$ and $X_{2}$ are associated with $T_{1}$ and $T_{5}$. The symmetries are also associated with $T_{2}$ and $T_{4}$ if $F_{1}(t)$ and $F_{3}(t)$ are constants, 


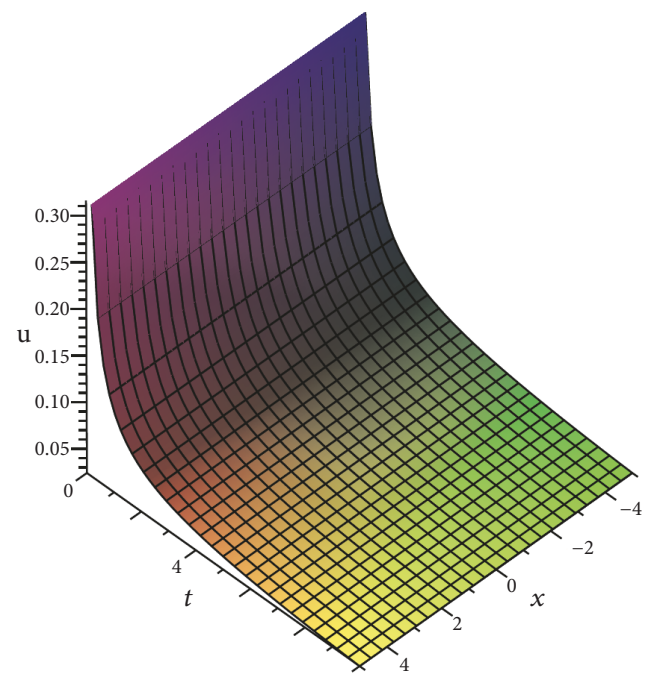

FIGURE 7: Solution $u$ given by (77) with $d_{8}=1$ and $r=1$.

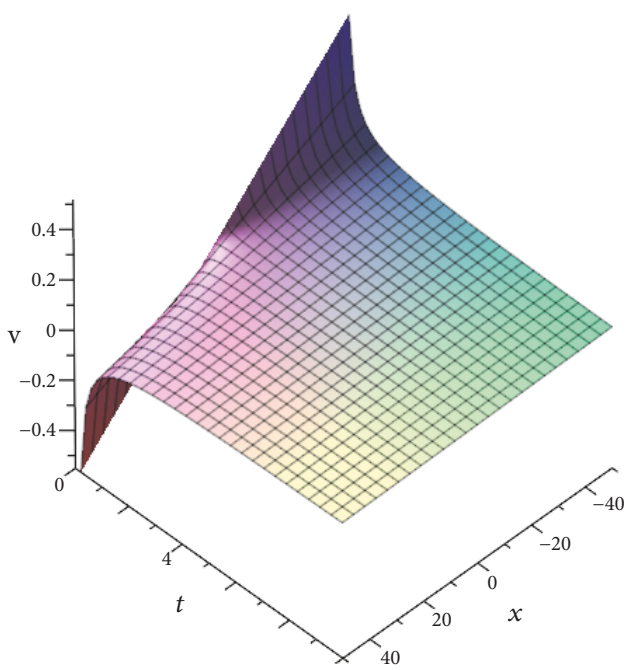

FigURE 8: Solution $v$ given by (77) with $k=1, d_{8}=1, d_{7}=1$, and $s=1$.

but in that case they coincide with $T_{1}$. Consequently, considering $T_{1}$ would be sufficient. The symmetry $X$ can then be expressed as

$$
\begin{gathered}
\frac{\partial}{\partial t}+\left(G_{1}(t)+\alpha G_{2}(t)\right) \frac{\partial}{\partial x}+\alpha \frac{\partial}{\partial y} \\
-\frac{1}{3 k}\left(G_{1}^{\prime}(t)+\alpha G_{2}^{\prime}(t)\right) \frac{\partial}{\partial v} .
\end{gathered}
$$

In similarity variables, we have

$$
\begin{aligned}
& a=-\int\left(G_{1}(t)+\alpha G_{2}(t)\right) d t+x, \\
& b=-\alpha t+y \\
& c=t
\end{aligned}
$$

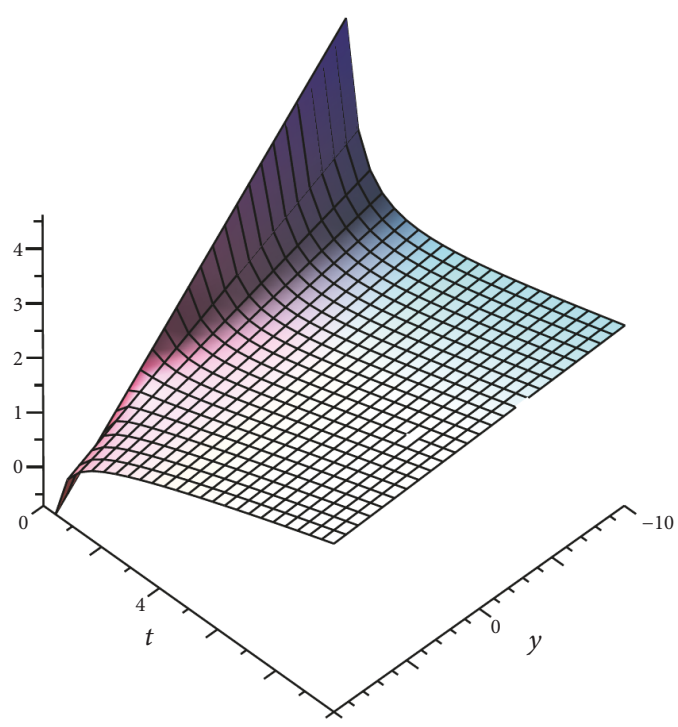

Figure 9: Solution $w$ given by (77) with $r=1, d_{9}=1$, and $q=1$.

$$
\begin{aligned}
& A(a, b)=\frac{G_{1}(t)+\alpha G_{2}(t)+3 v k}{3 k}, \\
& B(a, b)=w, \\
& C(a, b)=u,
\end{aligned}
$$

so that (18) gives

$$
\begin{aligned}
& T^{a}=-\left(G_{1}(t)+\alpha G_{2}(t)\right) T^{t}+T^{x}, \\
& T^{b}=-\alpha T^{t}+T^{y}, \\
& T^{c}=T^{t} .
\end{aligned}
$$

The reduced conserved vectors thus obtained are

$$
\begin{aligned}
& T_{1}^{a}=-k A C+s C+k C_{a a}, \\
& T_{1}^{b}=(q-\alpha) C-3 r C B+r C_{b b}, \\
& T_{5}^{a}=-B, \\
& T_{5}^{b}=C .
\end{aligned}
$$

The vectors $T_{1}$ and $T_{5}$ admit symmetries $\partial / \partial a$ and $\partial / \partial b$, from which further reduction is possible. Using combination of symmetries $\widetilde{X}=\partial / \partial a+\gamma(\partial / \partial b)$ and transforming it into new similarity variables yield

$$
\begin{aligned}
m & =a, \\
n & =-\gamma a+b, \\
L(n) & =C(a, b), \\
M(n) & =A(a, b), \\
N(n) & =B(a, b) .
\end{aligned}
$$


Again, from (18) it is straightforward to show that

$$
\begin{aligned}
& T^{n}=\gamma T^{a}-T^{b}, \\
& T^{m}=-T^{a},
\end{aligned}
$$

and system (3) satisfies

$$
D_{n} T^{n}=0
$$

Equations (84) and (85) give

$$
\begin{aligned}
& -k \gamma M L+s \gamma L+\left(k \gamma^{3}-r\right) L_{n n}-(q-\alpha) L+3 r L N \\
& \quad=d_{4}, \\
& \gamma N+L=d_{5}, \\
& M=-\gamma L+d_{6},
\end{aligned}
$$

where $d_{4}, d_{5}$, and $d_{6}$ are constants. System (86) reduces to Case 2.

\section{Results and Discussion}

Whereas the symmetry method only reduces the number of independent variables in the PDE involved, the double reduction method, in addition to the reduction of independent variables, reduces the order of the PDEs. The association of symmetries and conservation laws reduces the number of independent variables and the order of PDEs simultaneously. The association of a symmetry with more than one conservation law yields the same solutions (Case 2 of Sections 2 and 3 ), which are either implicit or in closed form. The association of scaling symmetry leads to double reduction whereas inversion symmetry yields a distinct solution to (1). The association of Lie symmetries and conserved vectors obtained in Section 3 involves arbitrary functions. The association of symmetries and conservation laws puts restriction on the form of these functions (as listed in Table 2). Under these restrictions, group invariant solutions are constructed. One solution is obtained using the relation between scaling symmetry and conservation law, while the other solutions are of travelling wave type.

Solutions (66), (67), (68), (69), (70), (74), (75), (76), and (77) are procured in terms of trigonometric functions using the sine-cosine method. An equivalent formulation to these solutions is given in [21] where the method of ansatz is employed to obtain dromion solutions in terms of exponential functions. In addition to the existing explicit solutions to (3), some new solutions including an implicit solution (64) and an explicit solution (77) to (3) have also been obtained. Moreover, we recuperated solution $\left(M_{3}, N_{3}, L_{3}\right)$ as reported in [17] using the combination of symmetries and conservation laws. This solution is identified as a dromion solution. In this article, we obtained three additional solutions $\left(M_{1}, N_{1}, L_{1}\right),\left(M_{2}, N_{2}, L_{2}\right)$, and $\left(M_{4}, N_{4}, L_{4}\right)$ of (3) which were not reported before.

Since the double reduction approach reduces the order of the PDE and the number of independent variables involved, it makes the numerical computations of solutions easier as compared to the application of numerical techniques to the original GNNV equation. This study provides a new way of constructing variety of exact solutions for partial differential equations by forming an association of the symmetry present with conserved vectors of the equation. This association is not only limited to the translational symmetries or transformation of the form $x-\alpha t$ and it may exist for a wide range of symmetries like rotations, dilatations, and inversions. In this article, we exploited the dilation symmetry of the systems to extract new exact solutions as stated above.

\section{Conclusion}

Double reduction of a PDE is a well-known technique which could be used when a conservation law is associated with Noether symmetry of Euler-Lagrange equation. However, in many cases, the PDE system does not admit the Lagrangian. Consequently, the existence of Noether symmetry remains out of question. In this work we considered systems of evolution equations which do not admit the Lagrangian formulation. We used the variational approach to construct conservation laws and their Lie symmetries. Double reduction was then employed by investigating the association between symmetry and conservation law. An association of scaling symmetry with a conserved vector was established. This had the consequence of integrating the differential equation further, which is significant since in many cases further integration of a differential equation is not possible if it is reduced by scaling or inversion symmetry. Several explicit solutions were also obtained which is a contribution of this paper.

\section{Data Availability}

No data were used to support this study.

\section{Conflicts of Interest}

The authors declare that they have no conflicts of interest.

\section{References}

[1] E. Noether, "Invariante variations probleme," Nacr Konig Gesell Wissen, Gottingen, Math-Phys Kl Heft, no. 2, pp. 235-257, 1918, (see Transport Theory and Statistical Physics, vol. 1, no. 3, pp. 186-207, 1971 for an English translation).

[2] A. H. Kara and F. M. Mahomed, "Noether-type symmetries and conservation laws via partial Lagrangians," Nonlinear Dynamics, vol. 45, no. 3-4, pp. 367-383, 2006.

[3] A. Sjöberg, "Double reduction of PDEs from the association of symmetries with conservation laws with applications," Applied Mathematics and Computation, vol. 184, no. 2, pp. 608-616, 2007.

[4] A. Sjoberg, "On double reductions from symmetries and conservation laws," Nonlinear Analysis: Real World Applications, vol. 10, no. 6, pp. 3472-3477, 2009.

[5] A. H. Bokhari, A. Y. Al-Dweik, F. D. Zaman, A. H. Kara, and F. M. Mahomed, "Generalization of the double reduction theory," 
Nonlinear Analysis: Real World Applications, vol. 11, no. 5, pp. 3763-3769, 2010.

[6] T. Congy, S. K. Ivanov, A. M. Kamchatnov, and N. Pavloff, "Evolution of initial discontinuities in the Riemann problem for the Kaup-Boussinesq equation with positive dispersion," Chaos: An Interdisciplinary Journal of Nonlinear Science, vol. 27, no. 8, 083107, 12 pages, 2017.

[7] A. M. Kamchatnov, Nonlinear Periodic Waves and Their Modulations: An Introductory Course, World Scientific, Singapore, 2000.

[8] X. Zheng, Y. Chen, and H. Zhang, "Generalized extended tanhfunction method and its application to $(1+1)$-dimensional dispersive long wave equation," Physics Letters A, vol. 311, no. 2-3, pp. 145-157, 2003.

[9] J. Zhou, L. Tian, and X. Fan, "Solitary-wave solutions to a dual equation of the Kaup-Boussinesq system," Nonlinear Analysis: Real World Applications, vol. 11, no. 4, pp. 3229-3235, 2010.

[10] L. J. Broer, "Approximate equations for long water waves," Applied Scientific Research, vol. 31, no. 5, pp. 377-395, 1975.

[11] D. J. Kaup, "Finding eigenvalue problems for solving nonlinear evolution equations," Progress of Theoretical and Experimental Physics, vol. 54, no. 1, pp. 72-78, 1975.

[12] L. Martinez, "Schrodinger spectral problems with energy dependent potentials as sources of nonlinear Hamiltonian evolution equations," Journal of Mathematical Physics, vol. 21, no. 9, pp. 2342-2349, 1980.

[13] B. A. Kupershmidt, "Mathematics of dispersive water waves," Communications in Mathematical Physics, vol. 99, no. 1, pp. 5173, 1985.

[14] A.-M. Wazwaz, "The generalized Kaup-Boussinesq equation: multiple soliton solutions," Waves in Random and Complex Media : Propagation, Scattering and Imaging, vol. 25, no. 4, pp. 473-481, 2015.

[15] B. Gao, "Symmetry analysis and explicit power series solutions of the Boussinesq-Whitham-BROer-Kaup equation," Waves in random and complex media : propagation, scattering and imaging., vol. 27, no. 4, pp. 700-710, 2017.

[16] C. Senthil Kumar, R. Radha, and M. Lakshmanan, "Trilinearization and localized coherent structures and periodic solutions for the $(2+1)$ dimensional K-dV and NNV equations," Chaos, Solitons and Fractals, vol. 39, no. 2, pp. 942-955, 2009.

[17] G. Xu and S. Deng, "Painlevé analysis, integrability and exact solutions for a $(2+1)$-dimensional generalized NizhnikNovikov-Veselov equation," The European Physical Journal Plus, vol. 131, no. 11, 2016.

[18] P. Albares, P. G. Estevez, R. Radha, and R. Saranya, "Lumps and rogue waves of generalized Nizhnik-NOVikov-Veselov equation," Nonlinear Dynamics, vol. 90, no. 4, pp. 2305-2315, 2017.

[19] D. G. Crighton, “Applications of KdV," Acta Applicandae Mathematicae, vol. 39, no. 1-3, pp. 39-67, 1995.

[20] M. Boiti, J. J. Leon, M. Manna, and F. Pempinelli, "On the spectral transform of a Korteweg-de Vries equation in two spatial dimensions," Inverse Problems, vol. 2, no. 3, pp. 271-279, 1986.

[21] R. Radha and M. Lakshmanan, "Singularity analysis and localized coherent structures in $(2+1)$ dimensional generalized Korteweg de Vries equations," Journal of Mathematical Physics, vol. 35, no. 9, pp. 4746-4756, 1994.

[22] S. T. Demiray and H. Bulut, "Soliton solutions of some nonlinear evolution problems by GKM," Neural Computing and Applications, 2017.
[23] C. Cattani, T. A. Sulaiman, H. M. Baskonus, and H. Bulut, "On the soliton solutions to the Nizhnik-Novikov-Veselov and the Drinfel'd-Sokolov systems," Optical and Quantum Electronics, vol. 50, no. 3, p. 138, 2018.

[24] R. Naz, I. L. Freire, and I. Naeem, "Comparison of different approaches to construct first integrals for ordinary differential equations," Abstract and Applied Analysis, vol. 2014, Article ID 978636, 15 pages, 2014.

[25] Zulfiqar Ali, Syed Husnine, and Imran Naeem, "Exact solutions of generalized modified boussinesq, kuramoto-sivashinsky, and camassa-holm equations via double reduction theory," Journal of Applied Mathematics, vol. 2013, Article ID 902128, 8 pages, 2013.

[26] H. Steudel, "Über die Zuordnung zwischen Invarianzeigenschaften und Erhaltungssatzen," Zeitschrift für Naturforschung, vol. 17A, pp. 129-132, 1962.

[27] P. J. Olver, Applications of Lie Groups to Differential Equations, vol. 107, Springer, New York, NY, USA, 2nd edition, 1993.

[28] A. M. Wazwaz, "A sine-cosine method for handling nonlinear wave equations," Mathematical and Computer Modelling, vol. 40, no. 5-6, pp. 499-508, 2004. 


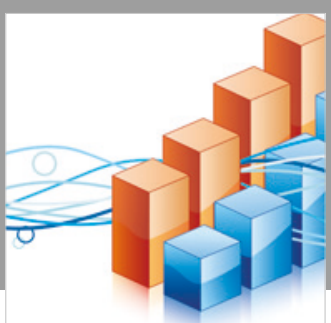

Advances in

Operations Research

\section{-n-m}
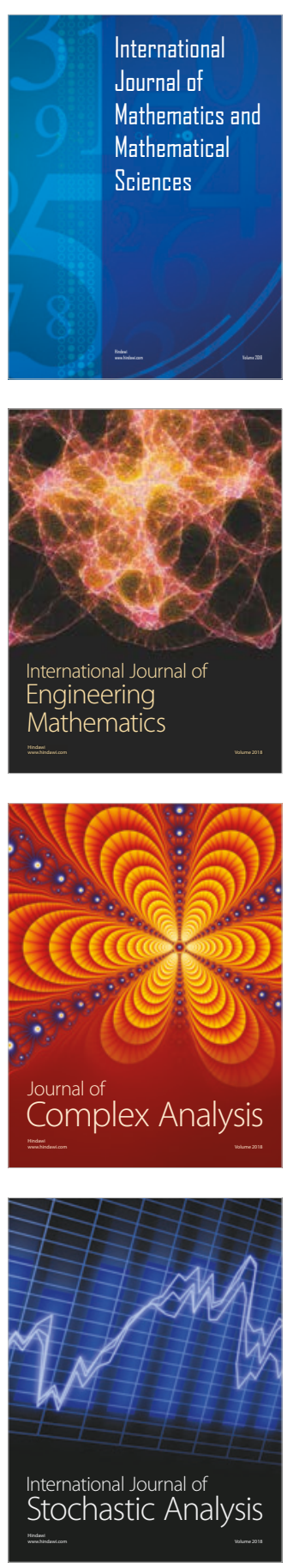
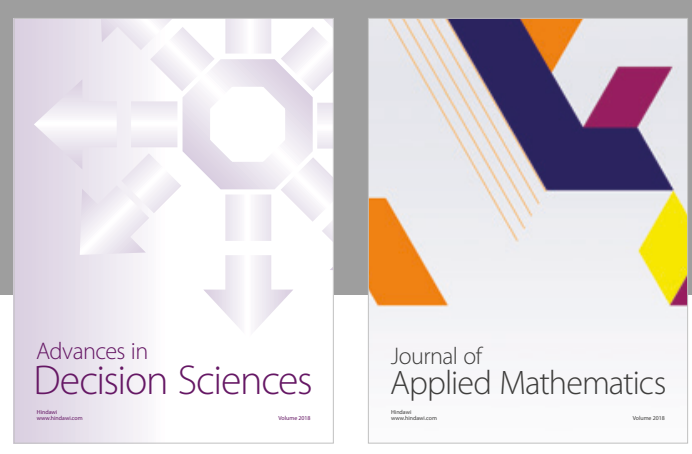

Journal of

Applied Mathematics
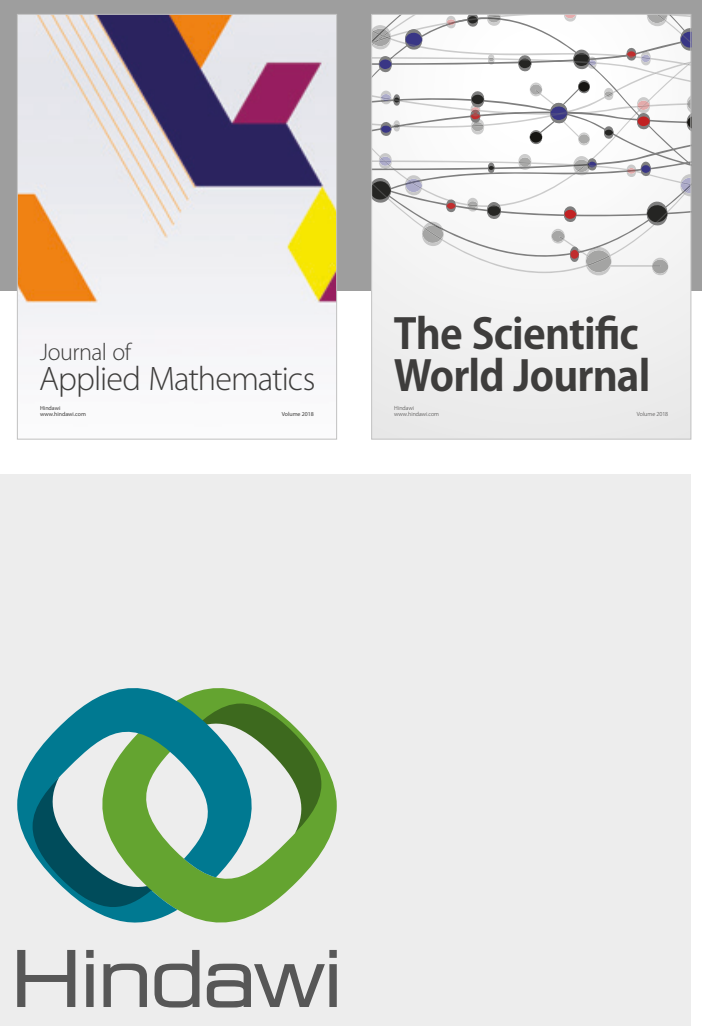

Submit your manuscripts at

www.hindawi.com

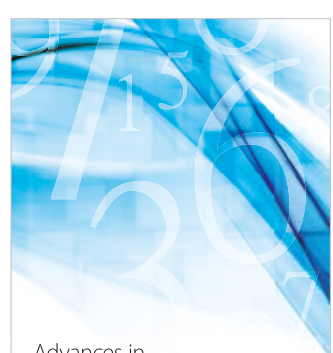

Advances in
Numerical Analysis
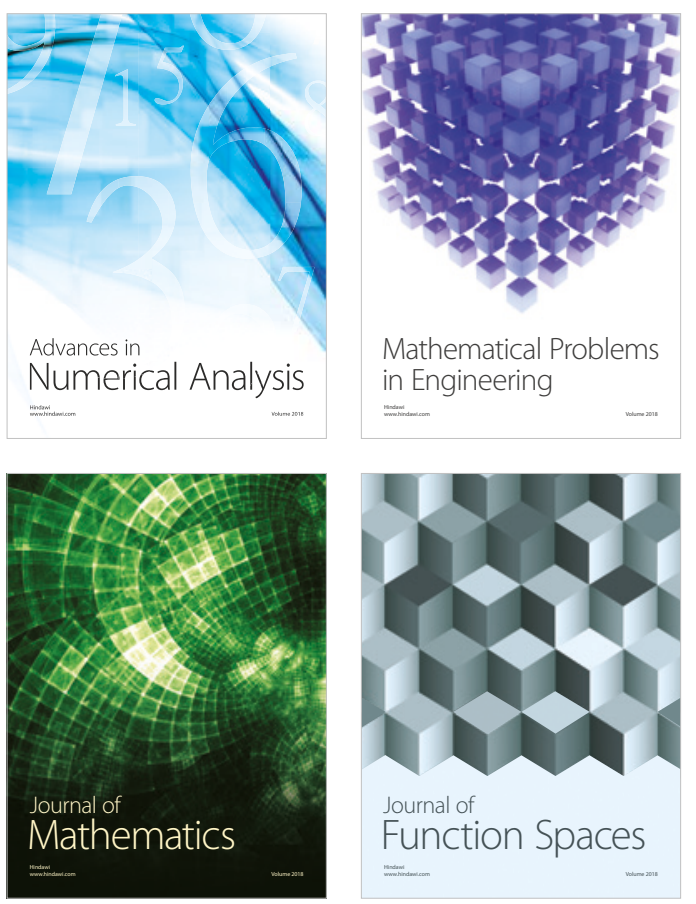

Mathematical Problems in Engineering

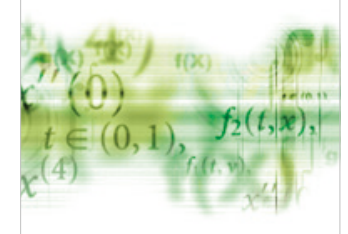

International Journal of

Differential Equations

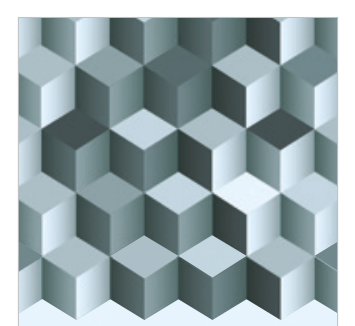

Journal of

Function Spaces

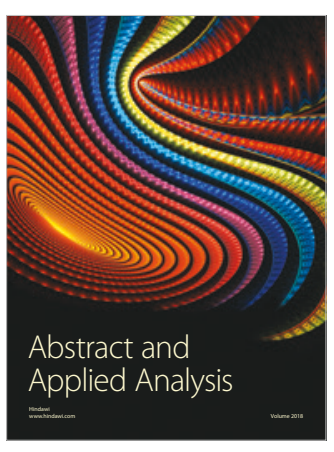

The Scientific

World Journal

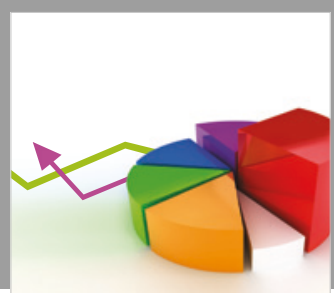

Journal of

Probability and Statistics
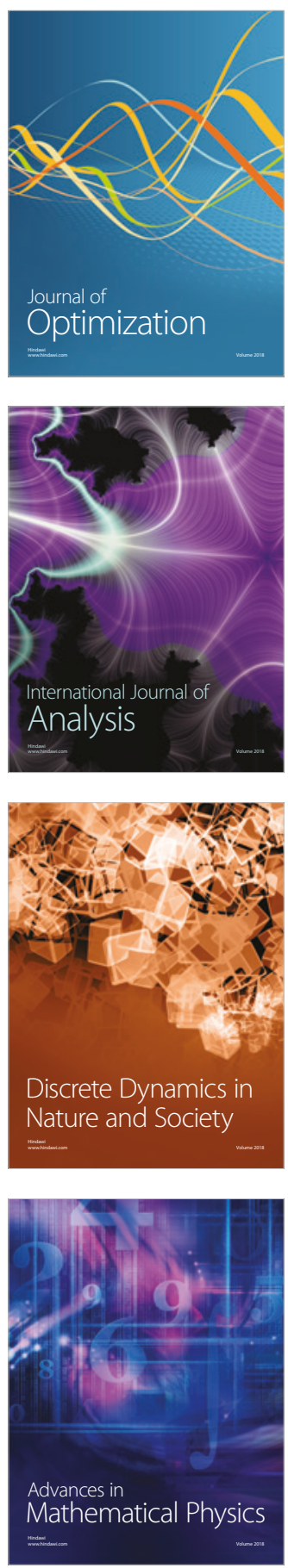